\title{
ELASTOMERIC RESERVOIR FOR MEMS-BASED TRANSDERMAL DRUG DELIVERY SYSTEMS
}

\author{
Cara T. Smith ${ }^{1,2}$, Pinghung Wei ${ }^{2,3}$, Mehran Mojarrad ${ }^{4}$, Michael Chiappetta ${ }^{4}$, Babak Ziaie ${ }^{2,3}$, and Masaru P. Rao ${ }^{5 *}$ \\ ${ }^{1}$ School of Mechanical Engineering, Purdue University, West Lafayette, Indiana, USA \\ ${ }^{2}$ Birck Nanotechnology Center, Purdue University, West Lafayette, Indiana, USA \\ ${ }^{3}$ School of Electrical and Computer Engineering, Purdue University, West Lafayette, Indiana, USA \\ ${ }^{4}$ Eli Lilly \& Company, Pharmaceutical Delivery Systems, Indianapolis, Indiana, USA \\ ${ }^{5}$ Department of Mechanical Engineering, University of California, Riverside, California, USA
}

\begin{abstract}
While MEMS-based drug delivery research often strives towards development of tightly-integrated device solutions, the authors present an alternate conception based upon a novel, decoupled drug reservoir approach. The salient features of this modular reservoir approach, which is the focus of this report, include an expandable PDMS enclosure within which the drug formulation is stored, an integrated rigid frame to provide mechanical integrity, and a vapour barrier to minimize diffusion-based losses. The design and fabrication of the reservoir is presented herein, as are favorable results from preliminary characterization of its fluid filling, retention, and extraction performance.
\end{abstract}

\section{INTRODUCTION}

The proposed reservoir is intended to serve as a key component in a MEMS-based transdermal drug delivery system that seeks to enhance patient acceptance and compliance with parenteral drug therapies. While such therapies are intended to improve both quality and duration of life for those with chronic disease, it is often found that patients do not follow their prescribed regimens, especially when frequent dosing is required [1]. For example, noncompliance is often observed in insulin therapy for the treatment of diabetes, which affects an estimated 170 million people worldwide [2]. While poor management of diabetes can lead to a variety of complications including vision impairment, heart disease, and neuropathy, upwards of $1 / 3$ of patients with type 2 diabetes nonetheless report aversion to insulin injections, due to fear of pain from needles, and $44 \%$ express lack of confidence in adhering to insulin therapy requirements [3]. Consequently, need exists for development of new delivery systems and strategies that address these issues, thus providing potential for increased patient compliance and overall health.
Top view

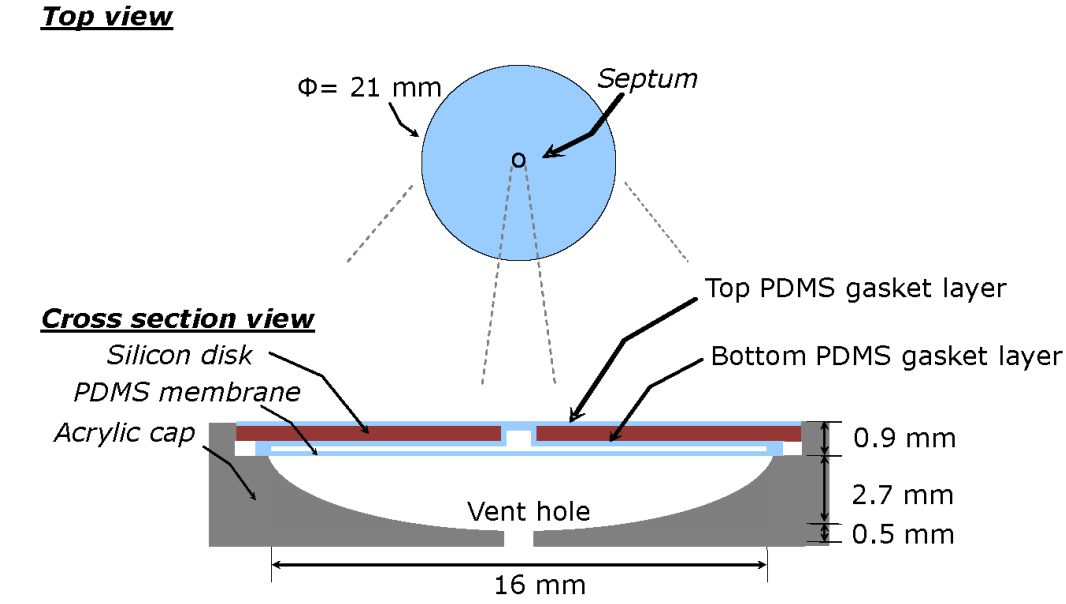

Figure 1: Reservoir schematic and filling process.
There currently exists a limited number of ways to treat diabetes with insulin, including administration via syringe and vial, pre-filled pen injectors, and insulin pumps [4]. Insulin pumps offer automated delivery, thus providing greater sense of freedom relative to manual injection, as well reduced demand upon the patient for self-management of their disease. However, insulin pumps are often found to be bulky, inconvenient to use, and hard to conceal. Miniaturized MEMS-based insulin pumps may provide a means for addressing these issues by offering greater convenience and discretion, due to decreased size. Moreover, when coupled with minimally invasive, microneedle-based, transdermal delivery, opportunity exists for mitigating needle anxiety and apprehension, as well as increasing ease of use.

These perceived benefits have driven significant interest in development of integrated MEMS-based components and systems for transdermal drug delivery. For example, of direct relevance to the current report, a number of compact, highly-integrated devices that rely on microneedle-based delivery have been recently reported [5-7]. However, only limited consideration has been given thus far to issues associated with filling and storage of drug formulations within such devices, particularly with regard to compatibility with conventional pharmaceutical practices. To this end, the authors propose that decoupling of the reservoir from the delivery system may, in fact, provide greater flexibility with respect to sterilization, plant-filling, delivery, mass production and manufacturing cost. Herein, we describe the design, fabrication, and preliminary characterization of a reservoir device based on this modular approach.

\section{RESERVOIR DESIGN}

The reservoir design, shown schematically in Figure 1, is comprised of a polydimethylsiloxane (PDMS) membrane and gasket enclosure with a centralized septum. An integrated rigid frame comprising of a silicon disk and vented acrylic cap provides
Cross section with fluid filled membrane

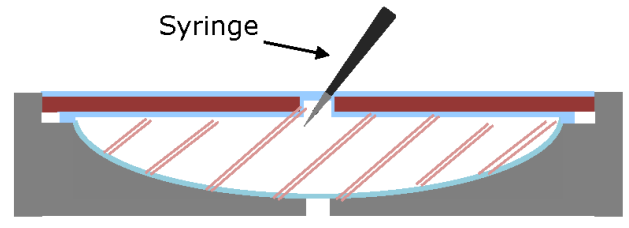

Cross section with polyimide seal

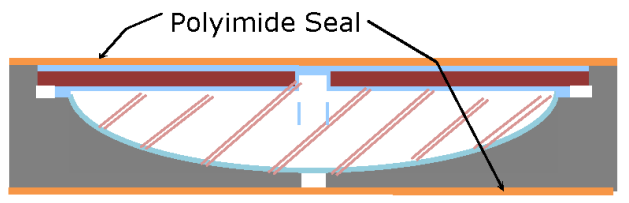


mechanical support and protection for the enclosure and its contents, as well as reliable interfacing with the aforementioned transdermal drug delivery system. The specific details of this system are not presented here, but include a hollow microneedle for septum penetration, a micropump for metered extraction of reservoir contents, and hollow microneedles for minimally invasive transdermal drug delivery.

The reservoir is intended to serve as a single-use cartridge that can be sterilized, filled, and stored long-term prior to assembly into the delivery system. Although the reservoir is currently filled manually via septum penetration with a syringe, its design is intended to provide future compatibility with conventional automated pharmaceutical vial filling operations. Final fill volume is fixed by the expansion of the elastic membrane to the inner dimensions of the cap. While the reservoir has been initially designed to contain $400 \mu$, other fill volumes can be easily accommodated with slight design modifications. After filling, the syringe is removed and the septum seals upon itself, thereby retaining the reservoir contents. Polyimide sealing layers are then applied to minimize diffusion-based fluid loss and ensure hermeticity.

The elastomeric and vapor-permeable nature of the PDMS membrane provides many advantageous features, including: a) membrane conformance to content volume; b) bubble-free deadfilling and drug storage; and c) mild internal pressurization to aid drug extraction $(\sim 10 \mathrm{kPa})$, due to strained membrane restoring force. Collectively, these features maximize reservoir content extraction, facilitate priming of the delivery system, and minimize overall reservoir size, complexity, and cost. Furthermore, the widespread use of silicone in pharmaceutical processing suggests potential for drug formulation stability during long-term storage, while membrane and cap transparency enables inspection for quality control and patient verification. Finally, reservoir decoupling allows sterilization without concerns for affecting other drug delivery system components, and also facilitates independent optimization of design, materials, and fabrication for each system component.

\section{MATERIALS AND METHODS \\ Fabrication}

The reservoir device is comprised of three primary components that are fabricated separately and then assembled into the final device. The first of these is the interface plate, which is fabricated by double-side molding of PDMS around a silicon disk. This interface plate serves as the fixed wall of the drug enclosure, as well as a rigid member that protects the contents and facilitates interfacing with the drug delivery system. The second component is the membrane, which is fabricated by PDMS molding and is then attached to the interface plate using uncured PDMS as a bonding adhesive. The membrane serves as the expandable wall of the drug enclosure, providing direct conformance of the enclosure to the content volume and thus minimizes dead volume and maximizes extraction efficiency. Finally, the third component is a vented acrylic cap, which is fabricated via conventional machining techniques. This cap serves to define the final fill volume of the enclosure and acts as a vapor barrier to prevent diffusion-based loss. It also provides protection for the membrane and enclosure contents and ensures that the device possesses sufficient rigidity to withstand handling during filling, packaging, and assembly into the drug delivery system by the patient.

Details of the reservoir fabrication process are schematically illustrated in Figure 2. The process begins with photolithographic patterning and deep etching of $100 \mathrm{~mm}$ diameter silicon wafers (Step 1) to create $500 \mu \mathrm{m}$ thick, $21 \mathrm{~mm}$ diameter disks that serve as backbone of the interface plate. Similar processes are then used to define silicon mold masters (Step 2a), which are then coated in a vacuum chamber with trichloro(3,3,3-trifluoro-propyl)silane to form a release layer. PDMS-based molds for the upper and lower faces of the interface plate are then made by pouring uncured elastomer (Sylgard 184, Dow Corning) into the silicon mold masters (Step 2b). The uncured molds are placed in a vacuum dessicator for $1 \mathrm{hr}$ to remove bubbles, followed by oven-curing for $30 \mathrm{~min}$ at $120{ }^{\circ} \mathrm{C}$. Upon removal from the mold masters, the PDMS-based molds are coated with Parylene C (thickness $\sim 2 \mu \mathrm{m}$ ), followed by silane coating. The former enhances the mechanical integrity of the molds, while the latter facilitates demolding.
1. Etch silicon disk with photolithography and DRIE etching
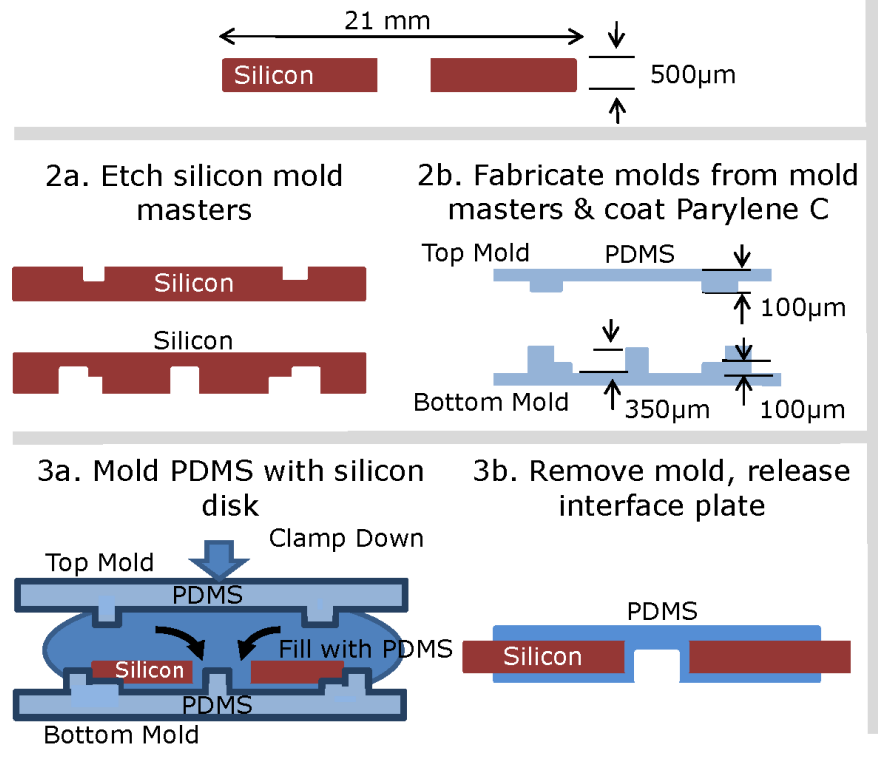

4a. Pattern SU8 on $4^{\prime \prime}$ silicon wafer

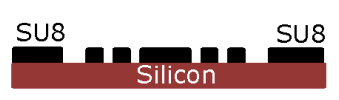

5a. Mold and cure PDMS

Flat Transparency
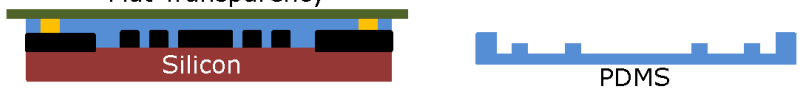

6. Bond interface plate to membrane with uncured PDMS

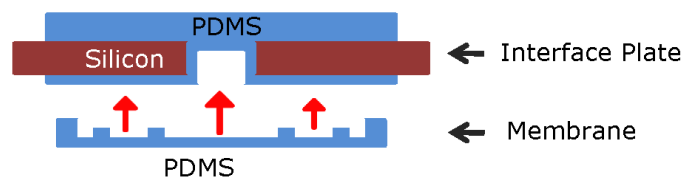

Figure 2: Reservoir fabrication process. 
Next, the interface plate is double-side molded by filling the bottom PDMS mold with uncured PDMS, followed by placement of the silicon disk, further filling with uncured PDMS, and placement of the upper PDMS mold (Step 3a). The stack is then mechanically clamped and oven cured for $30 \mathrm{~min}$ at $120{ }^{\circ} \mathrm{C}$. After curing is completed, the upper and lower molds are peeled away from the interface plate, and excess PDMS is trimmed from the boundaries (Step 3b).

Molding for the PDMS membrane is fabricated by photolithographic patterning of $100 \mu \mathrm{m}$ thick SU8 negative photoresist (SU8 2100, MicroChem Corp.) on a $100 \mathrm{~mm}$ diameter silicon wafer (Step 4a). This mold defines the membrane perimeter walls, as well as an array of cylindrical standoff posts $(50 \mu \mathrm{m}$ diameter, $100 \mu \mathrm{m}$ height, $100 \mu \mathrm{m}$ pitch $)$ on the inner face of the membrane, which serve to prevent irreversible adhesion of the membrane to the opposing enclosure surface. Small strips of polyimide tape (KPT2-4, 2 mil Kapton HN with 1.5 mil adhesive, Bertech-Kelex) are then placed at the perimeter of the mold (Step 4b) to serve as spacers that define the membrane thickness. The mold is then coated with a silane release layer.

The PDMS membrane is fabricated by filling the SU-8 membrane mold with uncured PDMS (Step 5a), followed by degassing in a vacuum dessicator for up to $1 \mathrm{hr}$. A standard transparency film is then placed on the uncured PDMS, followed by a blank silicon wafer, which serves as the upper part of the mold to ensure membrane planarity and thickness uniformity. The stack is then clamped and cured on a hot plate for four (4) hours at $60{ }^{\circ} \mathrm{C}$, followed by demolding (Step 5b). Once the membrane has been demolded, uncured PDMS is applied to the contacting surfaces at the perimeter to serve as an adhesive, and the membrane is manually aligned and brought into contact with the interface plate (Step 6). The reservoirs are then oven cured to complete the bonding process. Once cured, the acrylic cap is attached and the assembled reservoir device is filled by penetrating the septum of the interface plate with a fine gauge needle $(32 \mathrm{~g})$ attached to a syringe. Finally, the upper and lower faces of the device are sealed by applying polyimide tape.

\section{Fluid Retention}

The fluid retention performance of the filled reservoir devices was evaluated to provide preliminary indication of their potential for use in long-term storage applications. Retention performance was evaluated over a 3 week period using deionized water as a drug formulation analogue. Devices with and without the acrylic cap were tested, to determine the extent to which the caps mitigated vapor-based fluid loss through the membrane. Each device type was tested at three different storage temperatures to simulate a range of conditions that might be reasonably encountered in service, including refrigerated storage $\left(4{ }^{\circ} \mathrm{C}\right)$, room temperature storage $\left(21{ }^{\circ} \mathrm{C}\right)$, and extended exposure to body temperature $\left(37^{\circ} \mathrm{C}\right)$. Five $(5)$ devices of each type were tested and were weighed daily to determine the amount of fluid loss.

\section{Fluid Extraction}

The fluid extraction performance of the filled reservoir devices was evaluated to provide preliminary indication of their potential to efficiently deliver the majority of their contents. Extraction performance was evaluated by penetrating the septum of filled, uncapped devices with a 30 gage needle connected to a syringe. The contents of the reservoirs were then manually withdrawn by retracting the syringe plunger. Six (6) devices were tested and the extracted fluid volumes were compared to the initial filled volumes to determine extraction efficiency.

\section{RESULTS AND DISCUSSION}

Photographic images of completed and filled reservoir devices are shown in Figure 3. The integrity of the bonded interface between the membrane and the interface plate is apparent, as is the significant strain capability of the PDMS-based membrane. Although not shown here, additional experiments seeking to identify failure mechanisms associated with overfilling demonstrated that fill volume (in absence of the cap) was most often limited by delamination of the bonded interface between the membrane and the interface plate, typically at volumes well in excess of the design specification. There were no failures of devices filled to design specification, aside from those with clear manufacturing defects, e.g. incompletely bonded membrane or bubbles present in the membrane. This is as expected, since the design strain $(\sim 0.08)$ is well below the failure strain of PDMS (»0.5) [8], thus demonstrating the utility of this material for the current application with regard to mechanical performance.

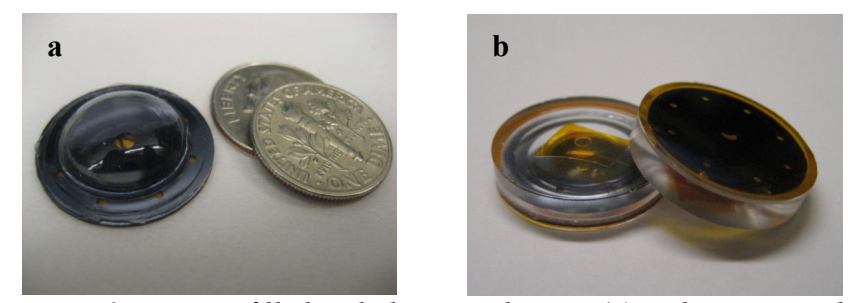

Figure 3: Devices filled with de-ionized water (a) without cap and (b) with cap and polyimide seals.

\section{Fluid Retention}

Fluid retention performance over a period of three (3) weeks under varying storage conditions for both capped and uncapped devices is shown in Figure 4. For each storage condition, devices without caps were observed to have higher fluid loss rates, as would be expected, given the high vapor permeability of PDMS. Moreover, in both device types, fluid loss rates were observed to increase with increasing temperature, as would be expected due to increased kinetics and thus increased atomic mobility. Finally, fluid loss rates were observed to be linear in all cases, regardless of internal fluid volume. The observation of negligible fluid loss in capped devices under refrigerated storage conditions is encouraging, since such storage conditions are required for many drug formulations. However, the increased losses observed at elevated storage temperatures indicate a need for further device refinement to ensure storage reliability under adverse conditions.

\section{Fluid Extraction}

Fluid extraction performance via manual withdrawal from six devices is shown in Table 1. High extraction efficiency is observed for all devices tested, ranging from $92.6 \%$ to $99.0 \%$ with average value of $96.1 \%(\mathrm{SD}=2.3)$. This can be primarily attributed to the minimal initial dead volume of the reservoir design, as well as the elasticity of the PDMS membrane, which precludes vacuuminduced inhibition of extraction by contraction during reservoir draining. Moreover, internal pressurization produced by the restoring force of the strained membrane further aids extraction. Although not shown here, additional experiments have demonstrated that measured internal pressures are in reasonable agreement with those predicted by theory. The demonstration of high extraction efficiency in these devices is encouraging, since 
this is a key factor in minimizing overall device size and waste of drug formulation.
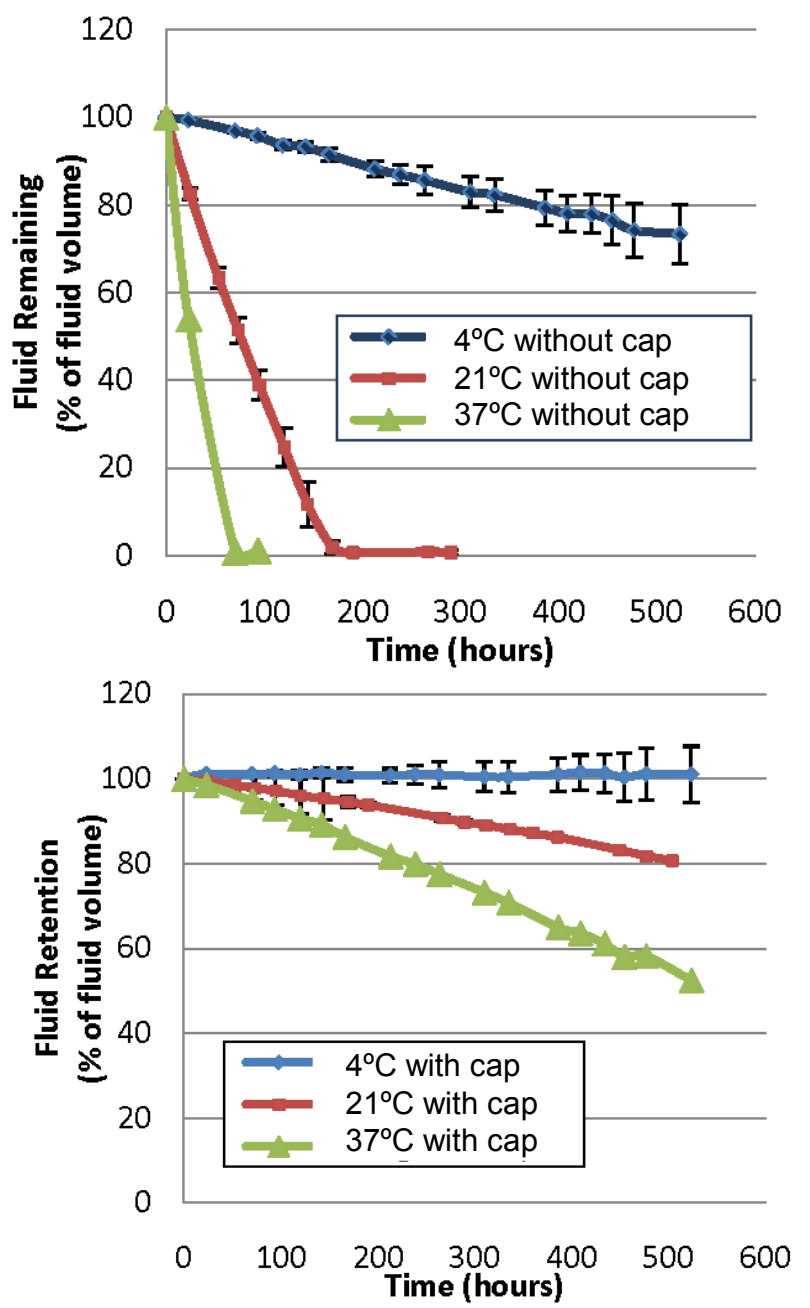

Figure 4: Fluid retention performance of reservoir devices stored at $4{ }^{\circ} \mathrm{C}, 21{ }^{\circ} \mathrm{C}$, and $37{ }^{\circ} \mathrm{C}$, without caps (top), and with caps (bottom) over a period of 3 weeks.

Table 1: Fluid extraction results.

\begin{tabular}{|c|c|c|c|}
\hline $\begin{array}{c}\text { Reservoir } \\
\text { Number }\end{array}$ & $\begin{array}{c}\text { Initial Liquid } \\
\text { Volume }(\mu \mathrm{L})\end{array}$ & $\begin{array}{c}\text { Extracted Liquid } \\
\text { Volume }(\mu \mathrm{L})\end{array}$ & $\begin{array}{c}\text { Liquid } \\
\text { Extraction }(\%)\end{array}$ \\
\hline 1 & 365 & 338 & 92.6 \\
\hline 2 & 374 & 364 & 97.3 \\
\hline 3 & 394 & 372 & 94.4 \\
\hline 4 & 372 & 357 & 96.0 \\
\hline 5 & 339 & 329 & 97.1 \\
\hline 6 & 386 & 382 & 99.0 \\
\hline Average Fluid Extraction (\%) & \multicolumn{3}{|c|}{2.3} \\
\hline \multicolumn{2}{|c|}{ Standard Deviation (\%) } & \multicolumn{3}{|c|}{} \\
\hline
\end{tabular}

\section{CONCLUSIONS}

The modular reservoirs described herein offer a unique approach for drug storage within MEMS-based drug delivery systems. They have demonstrated good delivery characteristics and long-term fluid storage potential, as evidenced by capability for reproducible delivery of over $95 \%$ of their contents, as well as negligible fluid loss under refrigerated storage conditions, which is required for many drug formulations. However, validation of sterility and stability of actual drug formulations within the reservoirs under pharmaceutically-relevant storage durations is needed. Moreover, device functionality has yet to be evaluated within the context of the transdermal drug delivery system within which the reservoir will operate. Finally, although the materials and fabrication process used for the reservoirs have proven sufficient for current needs, consideration of alternate materials and fabrication processes may be ultimately required to assure scalability to low-cost/high-volume manufacturing.

\section{AKNOWLEDGEMENTS}

The authors acknowledge Eli Lilly \& Company, Pharmaceutical Delivery Systems, for the funding of this research. The authors also acknowledge the staff of the Birck Nanotechnology Center at Purdue University for their help with equipment, supplies, and processes, in particular John Coy, Hassan Sharif, Geoff Gardner, Jeff Grau, and Ira Young.

\section{REFERENCES}

[1] J. Cramer, R. Rosenheck, G Kirk, W. Krol, J. Krystal, "Medication Compliance Feedback and Monitoring in a Clinical Trial: Predictors and Outcomes", Value in Health, 6 (2003), pp. 566-573.

[2] S. Wild, G. Roglic, A. Green, R. Sicree, H. King, "Global Prevalence of Diabetes: Estimates for the Year 2000 and Projections for 2030", Diabetes Care, 27 (2004), pp. $1047-$ 1053.

[3] W.H. Polonsky, S. Guzman, L. Villa-Caballero, S. Edelman, "Psychological Insulin Resistance in Patients With Type 2 Diabetes: The Scope of the Problem", Diabetes Care, 28 (2005), pp. 2543-2545.

[4] A.W. Brown, "Clinicians' Guide to Diabetes Gadgets and Gizmos", Clinical Diabetes, 26 (2008), pp. 66-71.

[5] B. Stoeber, D. Liepmann, A. Mokhtari, U.O. Häfeli, "In vivo evaluation of a microneedle-based miniature syringe for intradermal drug delivery", Biomedical Microdevices (2009).

[6] N. Roxhed, B. Samel, L. Nordquistt, P. Griss, G. Stemme, "Compact, Seamless Integration of Active Dosing and Actuation with Microneedles for Transdermal Drug Delivery", the IEEE 19th International conference on Micro Electro Mechanical Systems (MEMS 2006).

[7] S. Park, Y. Jang, H.C. Kim, K. Chun, "Fabrication of Drug Delivery System with Piezoelectric Micropump for Neural Probe", the 23rd International Technical Conference on Circuits/Systems, Computers and Communications (ITCCSCC 2008), pp. 1149 - 1152.

[8] F. Schneider, J. Draheim, R. Kamberger, U. Wallrabe, "Process and material properties of polydimethylsiloxane (PDMS) for Optical MEMS", Sensors and Actuators A, 151 (2009), pp. 95-99.

\section{CONTACT}

*M. P. Rao, tel: +1- 951-827-5870; mprao@engr.ucr.edu 